\title{
Trayectoria socio-técnica del sistema tecnológico feria de la agri- cultura familiar en la provincia del Neuquén
}

\section{Socio-technical trajectory of the family farming fair technological system in the province of Neuquén}

ARTÍCULO

\author{
Gustavo Federico Apablaza \\ Universidad Federal de la Integración Latinoamericana, Brasil. Contacto: federi- \\ co.apablaza@gmail.com
}

Recibido: abril de 2021

Aceptado: mayo de 2021

\section{Resumen}

El siguiente trabajo propone describir la trayectoria de las ferias pertenecientes a la agricultura familiar en la provincia de Neuquén. Estas pueden ser consideradas como parte de un sistema de producción, distribución e intercambio de bienes que se constituye como una tecnología de acceso a los mercados. Especialmente se estudia la trayectoria sociotécnica y las múltiples configuraciones que el sistema configuro estableciendo tres fases de despliegue. Ese proceso permitió situar al sistema en una trayectoria histórica concreta vinculada a contextos socio-económicos específicos. Este expresa complejas y heterogéneas relaciones sociales que hacen posible tanto la producción como el intercambio de bienes y servicios en el marco de alianzas, cada vez más complejas, que implicaron procesos de alineación y coordinación propios.

Palabras claves: Agricultura Familiar, Economía Social, Sistemas Tecnológicos Sociales.

\section{Abstract}

The following work proposes to reflect on the trajectory of family farming fairs in the province of Neuquén and Rio Negro. These can be considered as part of a system of production, distribution and exchange of goods that is constituted as a technology of access to markets. The socio-technical trajectory and the multiple configurations that it acquired are specially studied, establishing three deployment phases. This process made it possible to place the system on a specific historical trajectory linked to specific socioeconomic contexts. Understanding that they express complex and heterogeneous social relations that make the production and exchange of goods and services possible within the framework of 
increasingly complex alliances that implied their own alignment and coordination processes.

Keywords: Family Farm; solidarity social economy; social technology systems.

\section{Introducción}

En el presente trabajo se plantea la descripción de la trayectoria del sistema Feria de la Agricultura Familiar, a partir del análisis de las dinámicas socio técnicas que esa tecnología despliega en el Norte Patagónico, con énfasis en la provincia de Neuquén, a partir del uso de las herramientas analítico-conceptuales del enfoque socio-técnico.

Este proceso de análisis permite abrir la caja negra del sistema feria mostrando el funcionamiento de un sistema de producción, circulación e intercambio de bienes y servicios en el cual organizaciones y artefactos co-construyen estructuras de interacciones relacionados, desde su génesis, a problemas sociales más amplios. Siendo estas dinámicas y trayectorias las que generan procesos de persistencia, resistencia, innovación y cambio socio-técnico.

El presente artículo tiene por objetivo general el describir la trayectoria socio-técnica del sistema tecnológico feria de la agricultura familiar en la provincia de Neuquén. Para eso se establecen los siguientes objetivos específicos: Identificar a las ferias de la agricultura familiar como sistemas tecnológicos, y; especificar las fases de despliegue del sistema tecnológico feria de la agricultura familiar.

\section{Consideraciones Metodológicas:}

La estructura general de este artículo, y sus argumentaciones, se desprenden de la tesis de maestría Análisis de dinámicas socio-técnicas en Ferias de la Agricultura Familiar del Norte Patagónico: el caso de las Provincias de Río Negro y Neuquén. Para este trabajo se planteo la descripción del sistema Feria de la Agricultura Familiar, a partir del análisis de las dinámicas socio técnicas que esta tecnología despliega sustentada en el estudio de casos en la región del alto valle patagónico de las provincias de Río Negro y Neuquén. ${ }^{1}$

El presente trabajo posee un enfoque eminentemente cualitativo y se sustenta en dos bloques de herramientas metodológicas que permitieron la recolección y construcción de datos. En el primer bloque, se realizaron entrevistas semi-estructuradas a informantes clave, observaciones participantes y mapeo de actores. Esta instancia permitió comprehender la trayectoria del sistema tecnológico feria de la agricultura familiar con respecto a las diversas configuraciones que la misma fue adoptando hasta su estabilización.

1 Los casos relevados fueron: Feria de Artesanos y Productores de China Muerta, Feria de Parque España, Feria de Novella y Racedo. 
En el segundo bloque, la información recabada en el primer momento se enriqueció a partir de la incorporación de fuentes secundarias consistentes en publicaciones científicas e informes elaborados tanto por investigadores, organismos técnicos y de gestión (investigación y extensión) que permitieron una visión y contextualización mas acabada del objeto de estudio. Este análisis permitió la estructuración de cuadros explicativos subsidiados por dimensiones consideradas para el análisis de las trayectorias del sistema tecnológico feria de la agricultura familiar: relaciones usuario-productor; relaciones problema-solución; construcción de funcionamiento/no funcionamiento; racionalidades, y; políticas y estrategias.

Esta representación esquemática, facilita la compresión tanto de las configuraciones socio-técnicas como de posibles tensiones derivadas del sistema tecnológico feria de la agricultura familiar en el contexto específico del que forman parte, facilitando su comparación y posterior análisis.

\section{¿Por qué las ferias de la agricultura familiar pueden (y deben) ser consideradas un sistema tecnológico desde el campo Estudios Sociales de la Ciencia y la Tecnolo- gía?}

Situar el análisis de las ferias de la agricultura familiar desde el campo de los Estudios Sociales de la Tecnología (ESCT) implica abordar problemas relacionados con procesos de innovación, cambio tecnológico, generación de políticas públicas y procesos de desarrollo sustentable con inclusión social.

Este sistema socio-técnico de circulación no surgió de manera aislada. Es resultado de procesos endógenos de auto-organización que operaron al nivel de redes (Lepratte, 2011, p. 14), adoptando una configuración regional que, si bien se inscribe en un territorio, no hace necesariamente referencia a los aspectos de fronteras y jurisdicciones. El foco se encuentra en lo compartido en dimensiones como la tecnológica, política, productiva, ecológica y relacional, entre otras. Imprimiendo en el mismo un enfoque innovador para la resolución de problemas compartidos por amplios sectores.

Es en ese proceso de interacción donde se generan dinámicas al interior del sistema socio-técnico que implican el involucramiento de patrones de interacción de tecnologías y organizaciones, articulaciones y configuraciones socio técnicas (Villarreal, 2014). Esta dinámica incluye un conjunto de relaciones tecno-económicas y socio-políticas (Thomas, 2007).

Considerar a las Ferias de la Agricultura Familiar como un sistema tecnológico en el marco de la teoría constructivista de Ciencia, Tecnología y Sociedad (Thomas, Fressoli \& Lalouf, 2008, p. 74) requiere un proceso analítico que, al abrir la caja negra, revela que su 
construcción es producto de la compleja interrelación que se da entre individuos, grupos y tecnologías dando impronta a la particular característica socio-técnica de la misma.

A su vez es necesario no pensar en los problemas de desarrollo vinculados al sistema feria de la agricultura familiar a partir de los tipos de bienes y servicios que se intercambian, y sí en los funcionamientos que son deseables estabilizar en el marco de generación de dinámicas de inclusión con desarrollo sustentable. El pensar en las lógicas de funcionamiento permite a su vez el poder comprender y captar de una manera mucho más rica las relaciones problema-solución, teniendo noción de la dimensión de las dinámicas y la configuración en juego.

Para el presente trabajo fue tomado, como caso de estudio la Feria de Productores y Artesanos de China Muerta, de la localidad de Plottier, Provincia del Neuquén. Mientras que nos guiaremos analíticamente por los aportes de Winner (1986) quien plantea que es posible analizar y describir el concepto de tecnologías a partir del análisis de los diversos componentes presentes en un sistema tecnológico.

¿Cuáles son los componentes que giran en torno al sistema tecnológico feria de la agricultura familiar?

Considerando los aportes de Winner (1986) para describir los artefactos técnicos que se encuentran presentes en una feria, es relevante resaltar que los mismos son de vital importancia en relación con la dinámica problema-solución vinculada a los procesos de intercambio que se dan en su interior. Esto es así porque, en primer lugar ofrecen un soporte material (p. 28) que permite resolver, por un lado, el problema de la comercialización de excedentes y productos mediante un circuito corto de comercialización propio que posee un gran nivel de autonomía. En segundo lugar, contribuyen a reforzar posiciones políticas (p. 29) que promueven procesos de inclusión social en torno a la economía social y solidaria, el comercio justo, lo local, lo agroecológico, entre otros. La conformación, creación e implementación de instancias de intercambios (monetarios y no monetarios) alternativos implica y envuelve, necesariamente, procesos de política en su creación.

En relación a los procesos y plataformas tecnológicas necesarias para su funcionamiento los participantes debieron alinear y coordinar elementos heterogéneos en varios niveles: modos de producción y consumo; regulaciones locales y provinciales; conocimientos previos; recursos humanos y materiales, y; el soporte organizacional provisto por una cooperativa. Estos procesos y plataformas tecnológicas (Winner, 1986, p. 32) pusieron en juego habilidades, conocimientos, saberes, métodos, procedimientos y rutinas de los usuarios. Donde se dieron procesos de learning by doing o aprendizaje por la practica (Arrow, 1962) no solamente por parte de los usuarios (artesanos y productores) sino también de los actores técnicos e institucionales involucrados. 
Sobre los procesos organizacionales el hecho que los usuarios puedan contar con formas especificas de organizar el poder y la autoridad (Winner, 1986, p. 41) es de gran relevancia para el funcionamiento de la Feria. Con esto se hace referencia a tres elementos: El primero de ellos, con injerencia sobre los diversos procesos de gestión y organización que se dan en lo interno, como lo es el reglamento de la misma. El segundo, es la cooperativa como herramienta organizacional que aporta la institucionalidad de la feria ante otros actores. El tercero, hace referencia a la pertenencia a colectivos afines que proveen una estructura de soporte, metodológica y práctica. De esta forma los usuarios de la feria pertenecen a diversos colectivos: agroecológico, permacultura, economía social solidaria, feminismos y prácticas pedagógicas alternativas, entre otros.

\section{¿En qué contexto se estructura este Sistema?}

Durante la década de 1990 predominó, como única política de Estado, la lógica del ajuste permanente. Este proceso de retracción estatal generó una liberalización de todas las esferas de la vida, con la consecuente des-estructuración de relaciones tradicionales como el trabajo, la familia, la solidaridad entre trabajadores (Thomas y Becerra, 2012).

En la región patagónica se da una fuerte contracción de las fuentes de trabajo ligadas a la producción petrolera (fundamentalmente a partir de la privatización de YPF y de empresas provinciales) y frutícola (con la concentración de la industria), conjuntamente con la desarticulación de industrias incipientes como la cerámica y cementera, esto llevó a un marcado debilitamiento de los procesos de integración socio-económica y una creciente caída del salario social a partir del desmantelamiento de los restos del Estado de Bienestar que la dictadura no pudo, no quiso, o no se atrevió a eliminar.

Según el Censo Nacional Agropecuario del año 2002. En relación a la actividad vinculada a la agricultura familiar, entre 1988 y 2002, desaparecieron casi 100.000 unidades productivas, lo cual se tradujo en un aumento de la concentración de las propiedades en manos del agro-negocio y una expulsión de familias hacia los centros urbanos.

Esta concentración influyó en la participación global de los agricultores familiares en la producción global de alimentos en Argentina, pasando de un $52 \%$ a un $25 \%$, aproximadamente (Obschatko et al., 2007).

Siendo que en varios estudios se reconoce la importancia de la participación de la agricultura familiar tanto en su papel de la economía agropecuaria nacional como en su relevancia para la generación de dinámicas de desarrollo rural sustentable con enfoque territorial (Ramilo y Prividera, 2013; Obschatko et al., 2007; Soverna y Craviotti, 1999) estas modificaciones generaron un contexto en el que las Ferias de la Agricultura Familiar constituirán uno de los ejes de las relaciones problema/solución.

Frente a estas condiciones se produce, por parte de la población, un aumento reactivo de las estrategias de diversificación orientadas a garantizar el acceso a bienes y servi- 
cios diversos. Estas se basaron principalmente a partir de intercambios (monetarios y no monetarios) en diferentes canales con el fin de acceder a bienes básicos para garantizar la reproducción simple de la vida ya que se ponía en riesgo la accesibilidad y seguridad alimentaria de amplios sectores de la población.

A nivel regional se producen estrategias de movilidad espacial orientadas a la búsqueda de empleo; la lucha por la apropiación sobre los recursos productivos; la recuperación de espacios rurales a partir de la organización y de la socialización como alternativa para enfrentar la exclusión; la polarización de la distribución del ingreso y la consiguiente reducción del poder de compra de la clase media (Cassano et al., 2003, pp. 19-50; Svampa, 2000).

\section{Fases de despliegue del sistema tecnológico Feria de la agricultura familiar.}

La adopción de herramientas analitíco-comprensivas como lo es el análisis de las trayectoria socio-técnicas nos permiten explicar la manera misma en que los procesos sociales influencian en la forma y el contenido mismo de la tecnología- y viceversa-, a su vez nos permiten poder identificar el conjunto de patrones de interacción que se genera entre tecnologías, instituciones, políticas públicas, racionalidades y posicionamientos ideológicos de los actores ante un problema y las posibles soluciones sobre el mismo.

Desde allí resulta más adecuado hablar de lo socio-técnico (Thomas, 2006) en lugar de hablar de trayectorias tecnológicas. Detrás de todo artefacto hay una historia de tensiones entre productores y usuarios, políticas, regulaciones jurídicas, intereses económicos, culturas organizacionales entre otros. Esto vale tanto para las tecnologías de producto, como las de proceso o de organización (Jover, 2014).

Por otro lado, la noción de trayectoria socio-técnica nos permitirá comprender la coconstrucción de procesos sociales, productivos y organizacionales que se dieron en torno a las ferias de la agricultura familiar, identificar y situar las instituciones, las relaciones problema-solución planteadas por los grupos sociales relevantes, los procesos de construcción de funcionamiento y las racionalidades políticas y estrategias de los actores en juego. De esta manera es posible que podamos, a partir de tomar como eje de análisis el sistema feria de la agricultura familiar, ordenar las relaciones causales en secuencias temporales entre estos elementos heterogéneos.

En el caso de este trabajo, el punto de análisis, lo constituyen las estrategias de intercambio que se dan los agricultores familiares por intermedio de las Ferias de la Agricultura Familiar, la cual expresa una dinámica de co-construcción que se fue desplegando a partir de la dinámica problema-solución. 


\subsection{Primera Fase: Emergencia 1995-2002.}

A partir de la materialización de las consecuencias de las políticas liberales se genera, por parte de diversos actores, una serie de estrategias orientadas a garantizar la accesibilidad a los alimentos. Estas se materializan en iniciativas que promueven de dinámicas de intercambio de bienes y servicios alternativos hasta ese momento.

Aparece el mecanismo del trueque como una alternativa a esta situación, presentada desde sus comienzos como una forma de asociación libre, altamente consciente de valores y de relaciones solidarias, por medio de la cual se forman comunidades de prosumidores entendiendo como tales a quienes participan en el trueque, $y$, tienen la doble función de producción y ofrecer ciertos bienes y servicios, a la vez que demandan otros bienes y servicios dentro de la misma comunidad de intercambios.

Se da la vinculación de consumidores con oferentes en roles no diferenciados, como ocurre en los mercados predominantes que intercambiaban sus capacidades bajo la forma de bienes o servicios producidos y consumidos por ellos. Desde estas prácticas concretas, esta experiencia, cuestionaba la racionalidad mercantil que se había plasmado en prácticamente todas las estrategias de desarrollo implementadas hasta el momento desde las políticas públicas.

Según un estudio realizado por el Centro de Estudios Nueva Mayoría (Ovalles, 2002), en Argentina funcionaban durante el 2002 cerca de 5.000 clubes o nodos de trueque, tanto de la Red Global del Trueque, la Red del Trueque Solidario, como independientes. Del total, el 60\% (3.000 nodos) estaba en la provincia de Buenos Aires, el 18\% (900) en Santa Fe, el 4\% (208) en Capital, el 2\% (95) en Córdoba, el 1\% (65) en Mendoza y el $15 \%$ (732) restante en las demás provincias. Los integrantes según ese estudio ascenderían a 2,5 millones.

Denominamos a esta etapa Emergencia, tanto por el crecimiento explosivo que tuvo la experiencia del club del trueque (en un contexto donde la accesibilidad alimentaria se encontraba en serio riesgo) como por los aprendizajes organizacionales que aporto a los actores participantes de la misma. Esta experiencia tenía como objetivo final la generación de instancias de intercambio mediante la utilización de cuasi monedas poniendo como eje central el precio justo y la valorización de los saberes y haceres de los miembros. También en algunos casos se imponían los intercambios incluso sin la intervención de las cuasimoneda, pero esto era posible en clubes del trueque que poseían una cantidad limitada de participantes.

Estos espacios de intercambio no monetario funcionaron como una estrategia orientada a facilitar la circulación de bienes, en las ferias del trueque inicialmente confluyeron una gran diversidad de actores socio-productivos, la mayoría de ellos urbanos y periurbanos que no podían acceder o lo hacían en términos de intercambio muy desfavorables ante los mecanismos de comercialización mayorista y minorista. Entre estos actores se en- 
contraban: prestadores de servicios, agricultores, artesanos, revendedores, amas de casa, profesionales, estudiantes, obreros, entre otros. Estos actores compartían intereses comunes unidos por la necesidad de canalizar la oferta de bienes (alimentos, ropa, libros, maquinarias pequeñas, electrodomésticos y todo lo intercambiable) y servicios(arreglos eléctricos, servicios de peluquería, jardinería, computación, fletes, textiles, gasistas, albañiles, educativos, médicos, entre otros) que los participantes eran capaces de ofrecer, por un lado, y por otro los requerimientos de acceso a bienes y servicios, pero fundamentalmente de la posibilidad de acceder al consumo de alimentos.

Con la expansión de los Nodos del Club se profundizaron una serie de inconvenientes germinales del sistema (Cassano et al., 2003, pp. 107-237):

- La trazabilidad de la moneda emitida localmente con aquella emitida en otros nodos. Lo cual limitaba el acceso a los bienes y servicios inter-nodos ya que la construcción de la equivalencia suponía ampliar el nicho de confianza, siendo un condicionamiento estructural para la expansión la aceptación y equivalencia entre las monedas, lo cual suponía ampliar el ámbito de la confianza a cientos (luego miles) de nodos.

- El acceso a los insumos "extra-trueque". Muchos insumos necesarios para la producción de determinados bienes, fundamentalmente los de origen agropecuario, requieren dinero de curso legal para poder adquirirlos

- La vinculación con el Estado y la política. La cual se dio de forma contradictoria debido a diversas instancias e intentos de cooptación.

- El proceso organizativo del propio Club del Trueque. Carente de mecanismos de control específicos y compartidos entre todos los nodos.

En resumen, las condiciones socioeconómicas del país generaron una profunda presión sobre la experiencia del trueque que implicó un crecimiento explosivo de la escala de este. Si bien con esta estrategia se logró la posibilidad de que sectores empobrecidos y pobres puedan participar de instancias de intercambio (y con ello pudieran acceder a bienes y servicios básicos), también se reprodujeron mecanismos propios del marco tecnológico capitalista tanto en los comportamientos individuales como de las organizaciones (Cassano et al., 2003, p. 23).

Estas características problemáticas fueron estableciendo las bases para la introducción de las ferias libres o ferias francas en la región. Ellas, si bien se iniciaron a partir de intercambios con cuasi moneda, posteriormente se fueron tornando mixtas hasta finalmente abandonar los intercambios por cuasi moneda. 
En la región se creó en 1999 la Red Norpatagónica del Club del Trueque, donde participaban personas de distintas ciudades de Río Negro y Neuquén, ${ }^{2}$ generalmente en las ciudades de mayor población y cercanas a los Valles Irrigados (Neuquén Capital, Cipoletti, Centenario, Plottier. Compartiendo las mismas características y problemáticas ya mencionados.

En este punto los problemas identificados como prioritarios por parte de las organizaciones y del Estado fue establecer mecanismos que tanto aseguren la legitimidad de los productos vendidos como la necesidad de establecer patrones y mecanismos de control por parte de las autoridades para garantizar la sanidad de los alimentos garantizando la seguridad alimentaria.

En el caso de la provincia del Neuquén, se fue instalando la idea de que era necesaria la temprana intervención del Estado para regular las instancias de trueque (en muchas ciudades actualmente se continúa utilizando la denominación trueque como sinónimo de Feria) ya que a mediados del 2002 la mayoría de los intercambios eran monetarios.

En este punto de la trayectoria es visible la flexibilidad interpretativa en torno al Trueque/Feria como estrategia de intercambio, donde se hicieron presentes los diversos sentidos atribuidos al mismo por parte de los grupos sociales relevantes involucrados.

\footnotetext{
2 Datos al respecto pueden encontrarse en el diario Río Negro, domingo 21 de abril de 2002.
} 
Figura 1: Alianzas socio-técnicas del club del trueque. Fase emergencia 19952002.

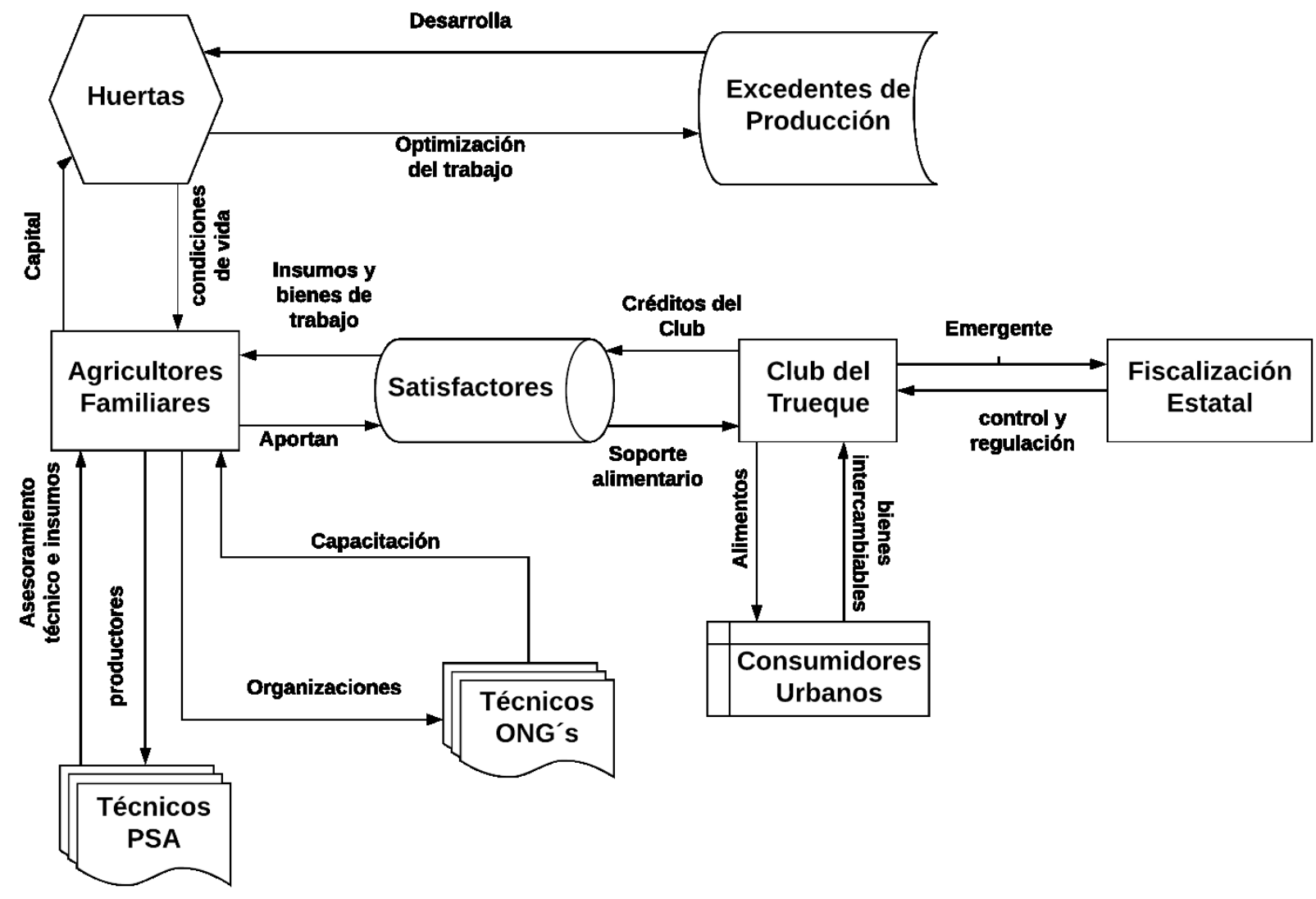

Fuente: Elaboración propia en base a entrevistas y fuentes secundarias.

Visibilizar e identificar la flexibilidad interpretativa de un artefacto implica exponer aquello que aparece como sólido, que nos muestra un objeto único e inequívoco (en este caso el trueque como estrategia de intercambio), pero en realidad aparecen tantos artefactos distintos cuanto sentidos sean atribuidos por los actores. Cada uno de estos artefactos, ocultos en lo que parece una única entidad, puede identificarse determinando los significados atribuidos por los grupos sociales relevantes en torno al problema de acceso a bienes y servicios, pero fundamentalmente, el acceso a alimentos.

Cuadro1: Fase Emergencia 1995-2002.

\begin{tabular}{|l|l|}
\hline $\begin{array}{l}\text { Relaciones Usuario- } \\
\text { Productor }\end{array}$ & Prosumidor, la relación es netamente entrópica, \\
\hline $\begin{array}{l}\text { Relaciones Problema- } \\
\text { Solución }\end{array}$ & Solución no-monetaria para la adquisición de bienes y servicios \\
\hline Construcción de Funciona- & Funciona a pequeña escala, soluciona problemas monetarios y \\
\hline
\end{tabular}


Divulgatio. Perfiles académicos de posgrado, Vol. 5, Número 14, 2021, 73-91 https://doi.org/10.48160/25913530di14.162

\begin{tabular}{|l|l|}
\hline miento/No Funcionamiento & cuestiona al mercado desde sus efectos. \\
\hline \multirow{2}{*}{ Racionalidades presentes } & $\begin{array}{l}\text { Intento de recomposición del tejido social, lucha contra la exclu- } \\
\text { sión. } \\
\text { Trueque como paliativo temporario } \\
\text { Resistencia }\end{array}$ \\
\hline Políticas y Estrategias & Trueque \\
& Visibilización del sector \\
& Alto grado de innovación \\
\hline
\end{tabular}

Fuente: Elaboración propia en base a entrevistas y fuentes secundarias.

2.2 Segunda fase: Negociación/disputa de sentidos (2003-2005)

A fines del año 2002 comenzaron a percibirse los primeros síntomas de reactivación económica, bajo nuevas reglas de juego (Panigo y Chena, 2011; Thomas y Becerra 2012):

- Un tipo de cambio múltiple que fomenta el desarrollo, de esta manera, cada sector obtiene un tipo de cambio.

- El impulso al consumo de bienes durables, en el que se torna competitiva la producción industrial local, a costa de los consumidores.

- La construcción y la industria sustitutiva también se vuelven rentables.

- La distribución del ingreso tiene posibilidades de mejorar de manera continua (aunque ello siempre supone una determinación y mediación social y política compleja),

- Progresiva incorporación de mano de obra por parte de sectores dinámicos con la absorción de mano de obra de sectores rurales y marginales hacia aquellos urbanos e industriales (pasando del 21,5\% en 2002 al 7,9 \% en 2011).

- Una activa intervención del estado en la economía, tanto en su papel de regulador como en la recuperación parcial de su rol productivo.

Con este contexto las condiciones de exclusión y marginalidad no desaparecen, pero comienzan a atenuarse, una gran proporción de la sociedad continúa con déficit para el acceso a alimentos, bienes y servicios a la vez que se profundizan los procesos de concentración a partir de las grandes cadenas de supermercados.

En este punto no estaba dada la estabilización de la estrategia trueque (donde priman los intercambios no monetarios) como una solución a este problema, pero si se van conformando consensos en torno a la instalación de Ferias como tecnología (donde pri- 
man los intercambios monetarios) siendo esta una solución de convergencia de varios actores: Municipios, Provincia, Agricultores Familiares, Medios de Comunicación, Comerciantes, Consumidores, entre otros. Se van dando un creciente grado de estabilización en torno al mecanismo organizacional de intercambio a utilizar la solución del problema.

Las Ferias paulatinamente se fueron transformando en lo que son actualmente en la región a partir de su consolidación producto tanto de la persistencia de los usuarios (productores-consumidores) como de la resistencia ante quienes se oponían a su permanencia y continuidad como herramienta de intercambio para los sectores desplazados.

En este proceso, actores que antiguamente se oponían a las ferias como tales (Municipios, Bromatologías, órganos de contralor y otros) paulatinamente van implementando políticas y acciones -explícitas o implícitas- orientadas a facilitar estos espacios de comercialización por parte de productores y artesanos.

Figura 2: Alianzas socio-técnicas del sistema tecnológico Feria de la Agricultura Familiar. Fase negociación/disputa de sentidos. 2003-2005.

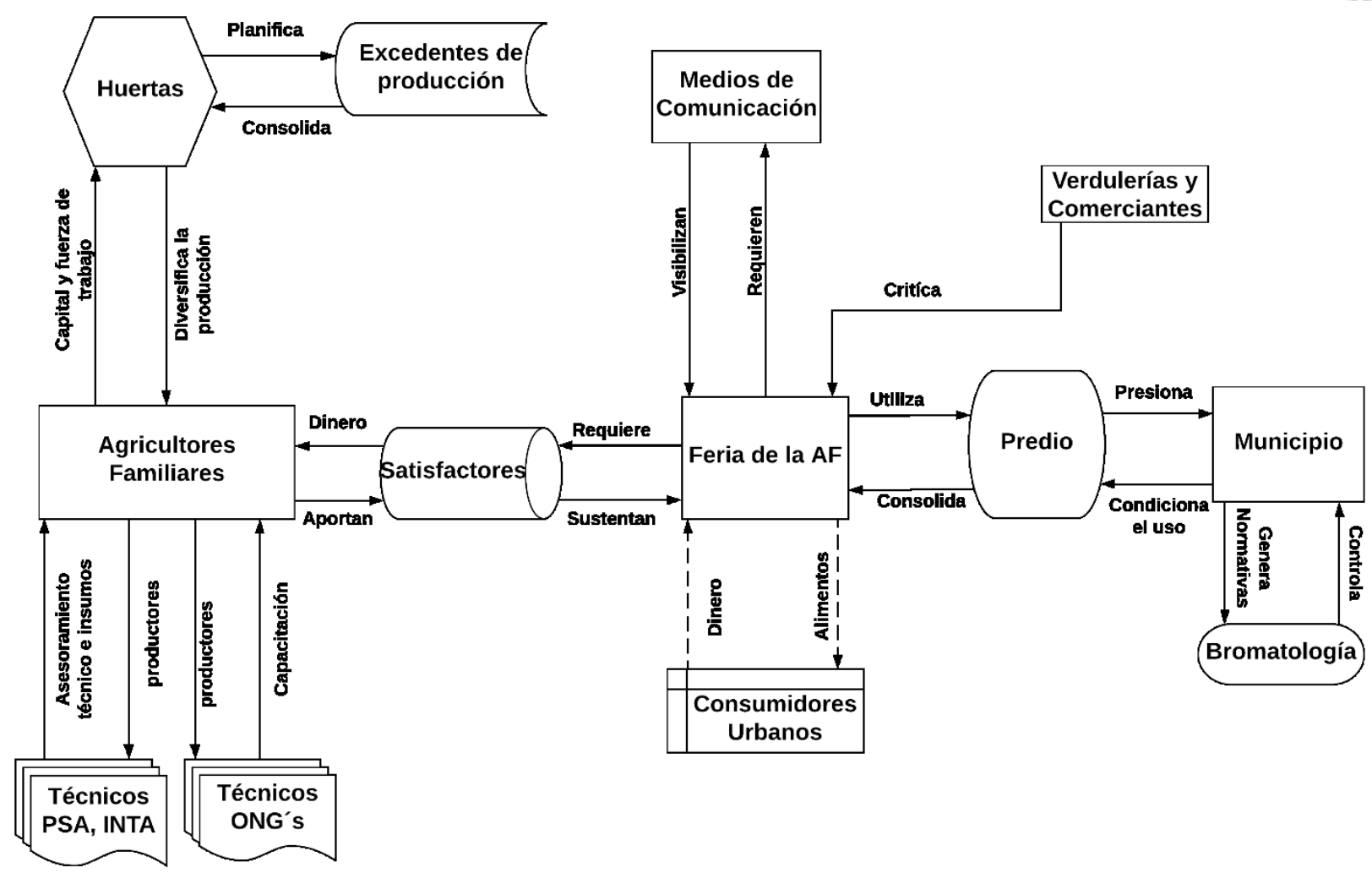

Fuente: Elaboración propia en base a entrevistas y fuentes secundarias.

Luego de un nivel inicial de controversia en torno a la orientación que las ferias deberían tener (si ser netamente de trueque; si ser mixtas; si netamente comerciales; si debían tener reventa, o; si solamente deberían participar productores locales o regionales), de los actores que deberían participar (agricultores familiares; estado municipal, provincial o na- 
cional; universidades; organizaciones de la sociedad civil; la iglesia católica, y; organizaciones de la economía social) y de los productos que deberían ofrecerse en ellas (artesanías, productos hortícolas, dulces y conservas, panificados, productos elaborados, carnes, pollos y huevos, productos usados, venta de productos importados) estas se estabilizaron como un instrumento para la comercialización (mediante intercambios monetarios) de los excedentes de pequeños productores familiares rurales y periurbanos de alimentos .

En relación a la continuidad de los intercambios no-monetarios se estabilizó el acuerdo a que el mismo debía ser principalmente mercantil-monetario, a pesar de que marginalmente pudiesen existir intercambios no monetarios basados en acuerdos del tipo feriante-feriante y feriante-consumidor.

Cuanto más homogéneos son los significados atribuidos a un artefacto, mayor es el grado de estabilización. Los distintos mecanismos de clausura que se dieron en torno a la Feria contribuyeron al establecimiento de una interpretación homogénea en torno a la misma como mecanismo per se de comercialización, lo cual conllevo paulatinamente a la desaparición de la flexibilidad interpretativa en torno a la orientación de la misma. Conjuntamente se fueron aplicando y consolidando nuevas prácticas vinculadas con la producción con enfoque agroecológico y sustentable (Becerra, 2015, p. 231).

Durante este periodo se consolidan 9 de las actuales Ferias en la provincia del Neuquén.

Cuadro 2: Fase Negociación/Disputa de sentidos 2003-2005

\begin{tabular}{|l|l|}
\hline $\begin{array}{l}\text { Relaciones } \\
\text { Productor }\end{array}$ & $\begin{array}{l}\text { Usuario- } \\
\text { nas al respecto, posee más visibilidad }\end{array}$ \\
\hline $\begin{array}{l}\text { Selaciones Problema- } \\
\text { Construcción de Funcio- } \\
\text { namiento/No Funciona- } \\
\text { miento }\end{array}$ & $\begin{array}{l}\text { Perduran situaciones de exclusión, mercado de trabajo en recomposicióng }) \\
\text { producción, consumo, intercambio y circulación } \\
\text { mercialización basadas en intercambios monetarios }\end{array}$ \\
\hline Racionalidades & Consolidar espacios generados, \\
& Persistencia \\
\hline Políticas y Estrategias & $\begin{array}{l}\text { Promoción y regulación de su expansión } \\
\text { Acompañamiento por parte de actores institucionales }\end{array}$ \\
\hline
\end{tabular}

Fuente: Elaboración propia en base a entrevistas y fuentes secundarias.

\subsection{Tercera fase: Consolidación y especialización (2006-2015).}


Durante ese periodo, el Estado argentino generó estrategias y políticas para la agricultura familiar con el objetivo de mejorar los aspectos tecnológicos, el acceso al financiamiento y al crédito, la adecuación de normativas en temáticas clave (tierras, comercialización y sanidad alimentaria) y la comercialización, fundamentalmente a través de la promoción de circuitos de proximidad o circuitos cortos de comercialización.

Estas acciones fueron coordinadas generalmente tanto con organizaciones sociales (asociaciones y cooperativas) como con gobiernos municipales, provinciales y nacionales. La negociación de los diferentes sentidos que los actores fueron imprimiendo al sistema feria fue colaborando con la estabilización del mismo, orientándose hacia el intercambio de productos producidos localmente (desde productos hortícolas, artesanías, dulces, conservas y licores entre otros) con lo que el perfil del sistema feria quedó con una clara dirección hacia lo productivo y con una función orientada a valorizar espacios de comercialización de los agricultores familiares de cada una de las localidades.

Figura 3: Alianza socio técnica del sistema tecnológico Feria de la Agricultura Familiar. Fase consolidación y especialización 2006-2015.

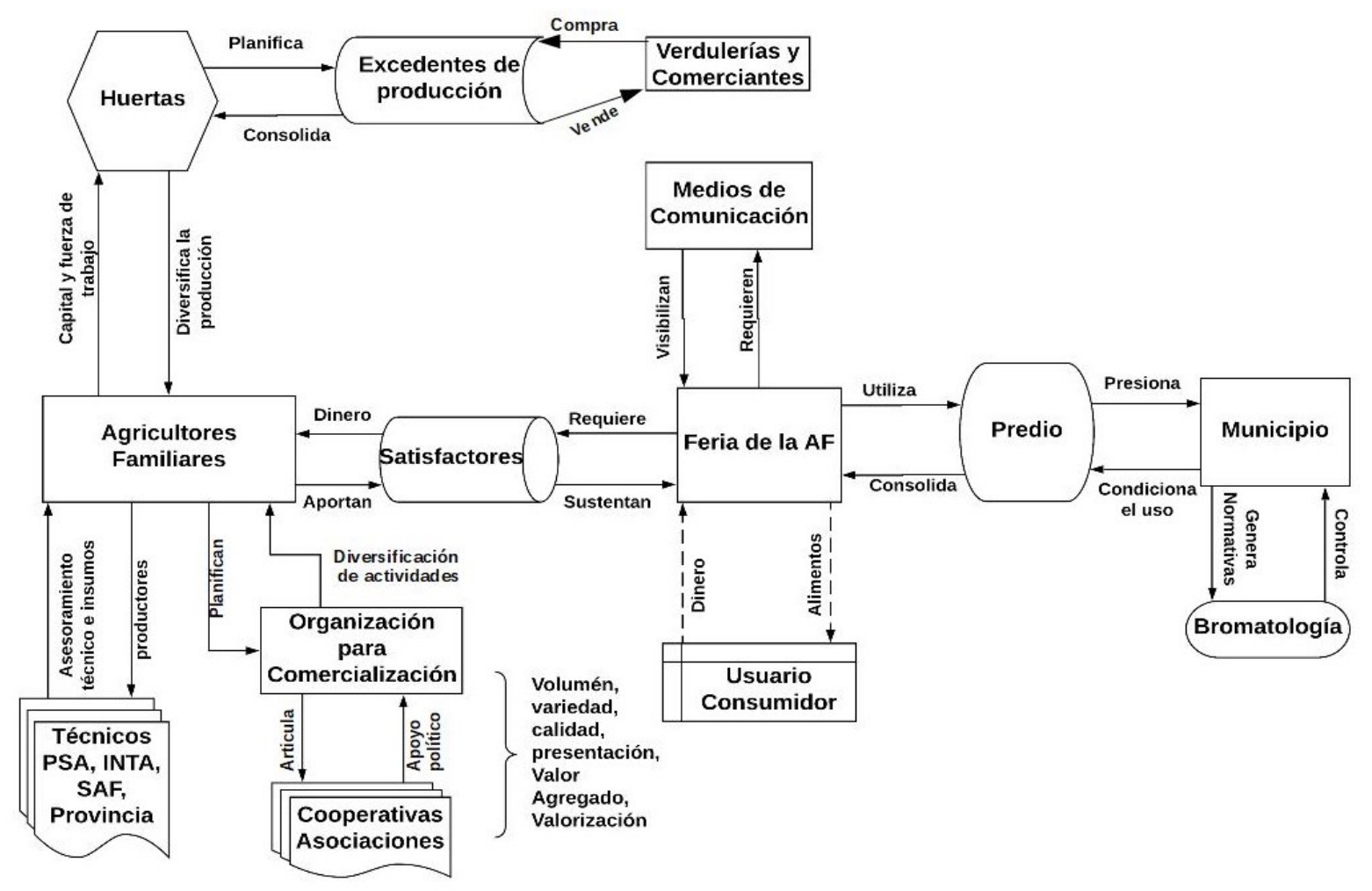

Fuente: Elaboración propia en base a entrevistas y fuentes secundarias.

Estos grupos sociales pusieron en juego su capital de trabajo y el capital que aportaba su conocimiento práctico o tácito (los dos capitales con los que contaban), como un elemento de construcción de poder y de resistencia socio-técnica (Valderrama y Jiménez, 2005; Garrido et al., 2011). En este proceso se fueron desarrollando, también, procesos de 
negociación/adecuación socio-técnica, en los que la adopción y adecuación del sistema feria fue posible. Por ejemplo, se desarrollaron capacitaciones sobre: aspectos zoofitosanitarios de la producción; manejo fitosanitario de cultivos; buenas prácticas agropecuarias y de manufactura; producción de alimentos agroecológicos y orgánicos; estrategias de comercialización; determinación de costos y precios; y, normativas, entre otros.

Fue así, que con el transcurso de los años en las provincias del Neuquén y Río Negro se fueron conformando un total de 38 ferias, las cuales se encuentran distribuidas a lo largo de la región de los valles protagónicos. Este es coincidente con el desarrollo de políticas de estado orientadas a promover las iniciativas de la agricultura familiar (Quintero y Alamo, 2013). Conjuntamente, desde 2004, la denominación agricultura familiar comienza a aparecer como tal en la agenda política de los organismos públicos argentinos (Paz et al. 2008) y consecuentemente se comienzan a articular diversas estrategias para la implementación de políticas públicas.

Si bien aquí se mencionan solamente 38 ferias, desde 1989 se han registrado en la región experiencias vinculadas a la creación de Ferias por parte de productores y organizaciones. No es objetivo del presente trabajo el analizar o registrar estas experiencias, pero si se considera importante indicar que constituyeron una instancia de aprendizaje socioproductivo para comunidad que contribuyó con saberes y prácticas para los agricultores y emprendedores; y, de aprendizaje institucional para los gobiernos locales que debieron regular y acompañar estos espacios alternativos de intercambio.

Del mismo modo es importante destacar cómo el sistema feria de la agricultura familiar incide cognitivamente sobre el usuario-consumidor. Este no es visto como un mero consumidor que accede a consumir para satisfacer sus necesidades reproductivas simples, sino que el mismo acto de consumir adquiere un nuevo sentido, el acto de consumo no es solo económico sino también político-ideológico.

Esta triplicidad (económica, política e ideológica) ligada al sistema ferias de la agricultura familiar inciden sobre las concepciones y orientaciones de desarrollo priorizando elementos inclusivos y principios solidarios ligados a prácticas agroecológicas, consumos responsables, economía social y solidaria, sistemas sustentables y producción orgánica por sobre la valorización del capital y la obtención de ganancias.

Cuadro 3: Fase Consolidación y Especialización 2006-2015

\begin{tabular}{|l|l|}
\hline Relaciones Usuario-Productor & $\begin{array}{l}\text { Interacción y educación. Multidimensional, educación del con- } \\
\text { sumidor, valorización }\end{array}$ \\
\hline Relaciones Problema-Solución & $\begin{array}{l}\text { Elección del espacio por sobre otras alternativas de consumo, } \\
\text { no solo prima la lógica mercantil en la resolución del problema }\end{array}$ \\
\hline
\end{tabular}




\begin{tabular}{|l|l|}
\hline $\begin{array}{l}\text { Construcción de Funcionamiento/No } \\
\text { Funcionamiento }\end{array}$ & $\begin{array}{l}\text { Funciona como espacio de consumo, de interacción social, } \\
\text { vinculado al desarrollo local. }\end{array}$ \\
\hline Racionalidades predominantes & $\begin{array}{l}\text { Mercantilistas y solidarias } \\
\text { Generación de renta y trabajo } \\
\text { Agregado de valor }\end{array}$ \\
\hline \multirow{2}{*}{ Políticas y Estrategias } & $\begin{array}{l}\text { Agregar valor } \\
\text { Andamiaje normativo }\end{array}$ \\
\hline
\end{tabular}

\section{Conclusiones:}

Durante el presente trabajo realizamos un recorrido que pretende analizar al sistema feria de la agricultura familiar como un sistema tecnológico de circulación de bienes y servicios.

Estas pueden ser consideradas como parte de un sistema de producción, distribución e intercambio de bienes que se constituye como una tecnología de acceso a los mercados.

Recurrir al enfoque socio-técnico permitió que pudiéramos abrir la caja negra del sistema Feria de la Agricultura Familiar. Ese proceso implicó analizar un sistema empírico con los conceptos analíticos que brinda el enfoque socio-técnico y permitieron poder situar al sistema en una trayectoria histórica concreta vinculada a contextos socioeconómicos específicos; entendiendo que existe una heterogeneidad de grupos y actores relevantes que inciden en la dinámica problema/solución

Las ferias de la agricultura familiar y el sistema tecnológico que ellas integran no son un mero artefacto aislado o un canal de comercialización, sino que las mismas expresan las complejas y heterogéneas relaciones sociales que hacen posible la producción y los intercambios de bienes y servicios en el marco de alianzas, cada vez más complejas, que implicaron procesos de alineación y coordinación propios.

Las condiciones socioeconómicas, sociopolíticas y organizacionales son determinantes para la orientación del sistema tecnológico. Para realizar el análisis fue fundamental el considerar la trayectoria histórica del sistema, y sin trayectoria no hay explicación que sea consistente para entender de qué manera el sistema se desenvuelve de manera autoorganizada para la resolución de un problema percibido por parte de productores y consumidores. 
Uno de los elementos que se destacan es que el sistema tecnológico feria de la agricultura familiar posee una relativa independencia de los actores políticos institucionales para sostenerse como tal. Y este hecho, en el que la participación de los usuarios y la sociedad fueron fundamental para la resolución del problema de acceso a los alimentos o de intercambio de los mismos por fuera de los canales hegemónicos de distribución, contribuye para la construcción de ciudadanía socio-técnica ya que el sistema fue sostenido por los propios usuarios.

El hecho que tanto agricultores familiares como consumidores hayan tenido que participar en los procesos de conformación, creación e implementación del sistema tecnológico feria requirió que deban desplegar intensos procesos de negociación de sentido tanto al interior de las organizaciones participantes como con las instituciones estatales implicadas las que inician procesos de desarrollo e implementación de política pública.

\section{Referencias Bibliográficas}

Arrow, K. (1962). The Economic Implications of Learning by Doing. The Review of Economic Studies, 29(3), 155-173. DOI: 10.2307/2295952

Becerra, L., (2015). Tecnología, Inclusión y Desarrollo. Hacia una teoría socio-técnica del desarrollo inclusivo. Tesis Doctoral. Facultad de Cs. Sociales, Universidad de Buenos Aires.

Cassano, D., Coraggio, J.L., Cortesi, J., Sabaté, A.M.F., Bombal, I.G., Hintze, S., Ilari, D., Krause, M., Laporte, L.N., La Serna, C. y Mance, E.A., (2003). Trueque y economía solidaria. Buenos Aires: Universidad Nacional de General Sarmiento.

Garrido, S., Lalouf, A. y Thomas, H., (2011). Resistencia y adecuación socio-técnica en los procesos de implementación de tecnologías. Los dispositivos solares en el secano de Lavalle. Revista AVERMA, 15.

Jover, J. N. (Ed.). (2014). Universidad, conocimiento, innovación y desarrollo local. La Habana: Editorial Universitaria Félix Varela.

Lepratte, L. (2011). Sistemas socio técnicos, innovación y desarrollo (26). MPRA. Recuperado en: https://mpra.ub.uni-muenchen.de/33559/1/MPRA_paper_33559.pdf.

Obschatko, E. D., MP Román, M. E., Pino, F. A., Morera, J. A., Oñoro, J. J., Villalobos, P., y A Paredes, L. A., (2007). Los pequeños productores en la República Argentina: importancia en la producción agropecuaria y en el empleo en base al censo nacional agropecuario 2002 (No. IICA 307.1412092 O14). Dirección de Desarrollo Agropecuario, Proyecto de Desarrollo de Pequeños Productores Agropecuarios, IICA, Secretaría de Agricultura, Ganadería, Pesca y Alimentación, Buenos Aires (Argentina).

Ovalles, E., (2002). Argentina es el país del mundo en el cual el fenómeno del trueque tiene mayor dimensión social. Centro de Estudios Nueva Mayoría. Recuperado en: http://www.nuevamayoria.com/invest/sociedad/cso080502.htm 
Panigo, D. y Chena, P., (2011). Del neo-mercantilismo al tipo de cambio múltiple para el desarrollo. Los dos modelos de la post-Convertibilidad. En Chena, P. I., Crovetto, N. E., \& Panigo, D. T. (Eds.). Ensayos en honor a Marcelo Diamand. Las raíces del nuevo modelo de desarrollo argentino y del pensamiento económico nacional (241-266). Buenos Aires: Miño y Dávila.

Paz, R., Jara, C. y Nazar, P., (2013). Economía Social y Agricultura Familiar. La experiencia de la Feria de Villa Río Hondo (Argentina). Cayapa. Revista Venezolana de Economía Social, 13(25).

Quintero, A. J. J., y Alamo, M. A., (2013). Análisis de las prácticas de intervención en la agricultura Familiar urbana. El programa pro-huerta en la ciudad de Bahía blanca. VII Jornadas Interdisciplinarias De Estudios Agrarios Y Agroindustriales. Centro Interdisciplinario de Estudios Agrarios (CIEA) de la Facultad de Ciencias Económicas de la UBA.

Ramilo, D., y Prividera, G. (2013). La Agricultura Familiar en la Argentina. Buenos Aires: INTA.

Soverna, S., \& Craviotti, C. (1999). Sistematización de estudios de casos de pobreza rural. Buenos Aires, Ministerio de Economía, Secretaría de Agricultura, Ganadería, Pesca y Alimentación. Dirección de Desarrollo Agropecuario. PROINDER. Serie documentos de formulación, (1).

Svampa, M. (ed.) (2000). Desde abajo: la transformación de las identidades sociales. Buenos Aires: Biblos.

Thomas, H. (2006). Trayectorias socio-técnicas y Estilos de cambio tecnológico en países subdesarrollados: la Resignificación de Tecnologías (Argentina, 1930-2006). XX Jornadas Argentinas de Historia Económica. Mar del Plata: Asociación Argentina de Historia Económica/Universidad de Quilmes.

Thomas, H., \& Becerra, L. (2012). Dinámicas tecno-económicas y generación de recursos humanos y cognitivos: Un análisis socio-técnico de la Argentina pre y post convertibilidad (2002-2011). Innovation RICEC, 3(2).

Thomas, H., \& Becerra, L. (2012). Dinámicas tecno-económicas y generación de recursos humanos y cognitivos: Un análisis socio-técnico de la Argentina pre y post convertibilidad (2002-2011). Innovation RICEC, 3(2).

Thomas, H., \& Fressoli, M. (2007). Repensar las Tecnologías Sociales: de las Tecnologías apropiadas a la Adecuación socio-técnica. In Congreso Latinoamericano y Caribeño de Ciencias Sociales-50 Aniversario de FLACSO, Quito (Vol. 29).

Thomas, H.; Fressoli, M. \& Lalouf, A. (2008). Presentación. Estudios sociales de la tecnología: ¿hay vida después del constructivismo? Redes, 14, 27, p. 59-76.

Valderrama, A. y Jiménez, J., (2005). Tecnología, cultura y resistencia. Revista de estudios sociales, (22). 
Villarreal, P. L., (2014). El proceso de construcción de una red socio técnica regional. El programa de control de carpocapsa en la fruticultura de las provincias de Río Negro y Neuquén, Argentina (Doctoral dissertation). Departamento de Economía y Ciencias Sociales. Universidad Politécnica de Valencia. (303p). Recuperado en: https://riunet.upv.es/handle/10251/36673

Winner, L. (1986). ¿Tienen política los artefactos?, en La Ballena y el Reactor. Gedisa Editorial, Barcelona.

Winner, L., Cardín, A. y Font, R., (1979). Tecnología autónoma: La técnica incontrolada como objeto del pensamiento político. Buenos Aires: Editorial Gustavo Gili. 